\title{
Confessione e RedenZIONE: L'ESEMPLARITÀ Parodica di Ser Ciappelletto e LAZARILLO DE TORMES ${ }^{1}$
}

\author{
Doris Nátia Cavallari*
}

ABSTRACT: Questo lavoro mette a confronto gli aspetti comuni della confessione parodica presente nel Lazarillo de Tormes (autore anonimo) e nella novella di Ser Ciappelletto, la prima novella del Decameron di Giovanni Boccaccio. L'analisi parte dal processo di carnevalizzazione della società, percettibile attraverso il discorso dei due protagonisti e dal luogo da essi occupato nella società rappresentata. La confessione parodica determina la rottura del pathos sociale, perché i protagonisti, proponendo la reinvenzione della propria storia attraverso il racconto di essa, rovesciano la loro posizione di antieroe all'interno del loro universo facendoli diventare uomini per bene, apparentemente adattati alla struttura morale, religiosa e politica del mondo rappresentato.

PAROLE-CHIAVE: Boccaccio; Decameron; Lazarillo di Tormes, confessione, parodia.

RESUMO: Este trabalho propõe uma comparação dos aspectos comuns da confissão paródica no Lazarillo de Tormes (de autor anônimo) e da novela de Ser Ciappelletto, a primeira do Decameron de Giovanni Boccaccio. A análise parte do processo de carnavalização da sociedade que se dá pelo discurso dos dois protagonistas. A confissão paródica determina a ruptura do pathos social pela

\footnotetext{
* Faculdade de Filosofia, Letras e Ciências Humanas da Universidade de São Paulo - USP

${ }^{1}$ Este trabalho foi apresentado no International conference "The monk, the priest, the nun", da Pen University da Philadelphia, EUA, com apoio da FAPESP, em março de 2013.
} 
reinvenção da própria história que inverte a posição do anti-herói e os faz figurar como homens de bem, aparentemente adaptados à estrutura moral, religiosa e política do mundo representado.

PALAVRAS-CHAVE: Lazarillo de Tormes; Decameron; Giovanni Boccaccio; confissão; paródia.

ABSTRACT: This paper proposes a comparison of the common aspects of parodic confession in Lazarillo de Tormes (anonymous author) and the novel of Ser Ciappelletto, the first of Giovanni Boccaccio's Decameron. The analysis starts by the carnivalization process of society that happens by the speech of the two protagonists. The parodic confession determines the rupture of the social pathos through the reinvention of their own history that reverses their position of anti-heroes and makes them appear as good men, apparently adapted to the moral religious and political structure fo the represented world.

KEY WORDS: Boccaccio; Decameron; Lazarillo di Tormes; confession; laugh; parody. 
Quanti ce ne devono essere a questo mondo che fuggono gli altri, solo perché non vedono se stessi.

Lazarillo de Tormes

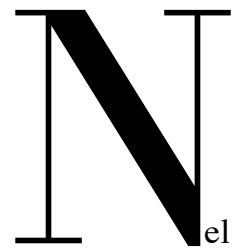

confessione come genere è più vicina al romanzo, perché è sempre una narrativa. Tuttavia, sottolinea due differenze fondamentali tra di esse, che sono il soggetto e il tempo, poiché nella confessione il soggetto è, allo stesso tempo, narratore e narrato, e la sua narrativa sarà sempre legata a una dimensione temporale, connessa alla sua stessa storia. Così, la confessione impedisce il girovagare per mondi immaginari, libertà invece concessa al testo letterario, che nasce dal mito e dalla favola, dato che l'artista "riproduce la creazione divina e crea un'eternità... virtualmente" (ZAMBRANO, 2000, p.42), mentre la confessione è legata alla realtà, al tempo della vita dell'uomo e esprime la visione della società di cui fa parte colui che si confessa.

La confessione, osserva ancora Zambrano, è legata all'oralità e richiede un linguaggio 
speciale "il linguaggio di qualcuno che non ha annullato la sua condizione di soggetto; è il linguaggio del soggetto in quanto tale. Non sono i suoi sentimenti, né i suoi desideri, né le sue speranze; sono semplicemente i suoi sforzi di essere. È un atto in cui il soggetto si rivela a se stesso perché ha orrore del suo essere a metà e confuso" (ZAMBRANO, 2000, p.43). La confessione è, quindi, una necessità intima di rivelarsi e di "ri-significare" la propria esistenza, analizzando gli errori e cercando di correggerli per prepararsi all'avvenire come un uomo rinnovato. Il soggetto, quindi, si confessa per mantenere un legame con la realtà tramite il rendiconto della propria storia fatta di difetti, illusioni, frustrazioni e dell'impossibilità di raggiungere quell'ideale di perfezione che pervade la soggettività sociale di ogni comunità umana. Questi sono aspetti comuni che conformano il millenario immaginario cristiano, che ritiene la contrizione e la confessione siano l'unica speranza di redenzione e di pace. Vista da questo punto di vista, la confessione diventa la forma più pura e diretta dell'interazione dell' "io" con l'altro che, per ragioni di fede, è disposto a sentire e a perdonare; è un momento catartico della vita, nella quale l'uomo fa un resoconto della sua storia e pensa anche alla futura morte, o soltanto alla transitorietà dell'esistenza terrena.

Se con Zambrano consideriamo gli aspetti narrativi della confessione e quelli della letteratura e del romanzo, possiamo pensare a due testi molto particolari dell'inizio della tradizione narrativa moderna, dato il rovescio parodico proposto dai narratori. Parlo della confessione di Ser Ciappelletto nella novella che apre il Decameron di Giovanni Boccaccio e della lettera confessionale a "vossignoria" di Lazarillo de Tormes, testo di autore anonimo, pubblicato nel 1554, e considerato la prima opera della picaresca spagnola e anche la prima forma aurorale del romanzo moderno.

Il Decameron, come si sa, è stato scritto tra il 1348 e 1352, nel periodo della terribile peste nera a Firenze, descritta in dettagli dal narratore, che insiste sui particolari della dissoluzione dei valori e dell'impossibilità di una pacifica coesistenza civile, o "quest'orrido cominciamento", per dare più rilievo al piacere e alla letizia che li seguiranno. La proposta di racconti lieti resterà a carico di un gruppo di dieci giovani che, per fuggire dal caos e dalla paura della morte, scappa dalla città e, soggiornando in un "locus amoenous", si propone di raccontare storie a turno, nelle ore più calde del giorno.

Ma il tutto è scatenato dalla peste e dalla morte. Lo storiografo Jean Delumeau, nel testo La paura in Occidente, osserva che quando c'è la guerra o la peste si verifica una sorta di abolizione della morte personalizzata (2006, p. 123), ossia, non c’è più spazio per le lacrime, 
per i ricordi, per la veglia e il corteo funebre. La peste costringe alla solitudine nella malattia e nella morte. Se pensiamo con Delumeau, possiamo capire perché, per riorganizzare il mondo tramite la narrativa, la prima novella del Decameron abbia come tema la morte del protagonista, ser Ciappelletto, presentato al momento in cui è moribondo, in una terra straniera e praticamente sconosciuto da tutti. Però, al contrario di quello che avveniva nella Firenze colpita dalla peste, il protagonista avrà diritto a un corteo funebre completo, con tutte le attenzioni rivolte alla sua persona e alla sua dignità, tanto onorata da un ingenuo frate che crede alle sue parole e lo considera perfino un santuomo.

Ma prima di arrivare a questo momento di "gloria" per la memoria del morto, il narratore della prima novella, Panfilo, ci presenta in poche parole la biografia di Ciappelletto e conclude dicendo che egli era il peggiore uomo forse che mai nascesse (BOCCACCIO, Decameron, 2010, p.47). Infatti, il notaio che amava la falsità, l'assassinio, la menzogna, era bestemmiatore, sodomita, golosissimo e bevitore grande, giuocatore e mettitor di malvagi dadi (p. 48), è il personaggio sintesi dell'arte dell'inganno o dell'arte come inganno, come osserva il critico Guido Almansi.

La novella di Ciappelletto è stutturata sulla base di una breve presentazione (Ciappelletto come uomo malvagio), di una lunghissima confessione falsa e di un brevissimo epilogo. La falsa confessione - fatta da Ciappelletto per salvare la reputazione di due fratelli usurai che lo ospitavano e lo curavano nella malattia e che avrebbero avuto dei problemi dopo la sua morte se fosse avvenuta senza la benedizione di un religioso - è l'ultimo atto di un antieroe, di un uomo che "avea grandissima vergogna quando uno de' suoi strumenti (come che pochi ne facesse) fosse altro che falso trovato" (BOCCACCIO, 2010, p.48).

La falsa confessione alle porte della morte non spaventa, quindi, il malvivente, anzi, Ser Ciappelletto ha come ultima, divertente e paradossale sfida quella di burlare un santo uomo, conoscitore delle sacre scritture. Ed effettivamente il protagonista riesce, con grande abilità discorsiva, a re-inventare la propria storia e a convincere il frate della sua beata personalità. Il culmine di questa "rilettura" della storia è il fatto di venir poi adorato dal popolo locale e di essere proclamato Santo, e, da quel momento in poi, esser conosciuto come San Ciappelletto.

Il grande menzognero e ladro utilizza il discorso di uomo dabbene, la "parola internamente convincente", per dirla con Bachtin, quella che è "semipropria, semialtrui" e quindi ottiene il riscontro atteso dallo ascoltatore che l'accetta pienamente, perché la sente come unespressione che potrebbe essere sua. 
A partire dell'uso di un linguaggio adeguato alla situazione, il protagonista boccacciano si assicura l'accettazione popolare e tutti gli omaggi che un morto illustre si merita. Abbiamo, quindi, il ritorno della "morte personalizzata", con la realizzazione di un vero e proprio rituale del lutto, quello stesso rituale che durante il periodo della peste nera era stato sospeso. È vero, però, che in un certo senso questo rituale dovrebbe essere considerato sconsacrato, giacché si tratta di un menzognero di cui il lettore conosce la vera storia. Eppure, il narratore della novella, Panfilo, concluderà che la benevolenza (benignità) del Signore deve considerare molto di più la purità della fede - quella del popolo, si capisce - e la sì fatta contrizione della confessione, fatta così bene e in modo convincente, che fa sembrare beata la vita scellerata del notaio. Possiamo pensare che il Dio di Boccaccio darebbe, così, più attenzione alla capacità creativa del verbo, molto somigliante al Verbo, che non alle azioni umane oppure che prenderebbe in considerazione il libero arbitrio di Ciappelletto, il quale, essendo fedele alla propria scelta esistenziale di ingannare il prossimo, mantiene la sua "integrità" di uomo malvagio e, allo stesso tempo, compie una buona azione e salva i suoi compagni da un grosso problema. La prima novella del Decameron, opera totalmente fondata sull'ironia, spezza definitivamente la rigidità del mondo medievale e offre soluzioni nuove o inaspettate.

Quando scrive su Rabelais, Bachtin si trattiene a lungo sulla questione del riso nel medioevo, sulla quale cercherò di fare un breve commento. Infatti, il riso medievale avveniva nei momenti di festa che - afferma Bachtin - "era un'interruzione temporanea di tutto il sistema ufficiale, con i suoi divieti e le sue barriere gerarchiche" (1975, p. 100). Il teorico russo commenta che, tra le caratteristiche di questa festa carnevalesca, c'era la libertà, cioè la possibilità di esprimere "la verità popolare non ufficiale" che era contraria alla serietà ufficiale, poiché

L'uomo medievale percepiva chiaramente nel riso la vittoria sulla paura"... non soltanto come vittoria sulla mistica ("paura divina”), ma sulla paura davanti alle forze della natura, soprattutto come vittoria sulla paura morale, che incatena, opprime e offusca la coscienza dell'uomo ("tabu" e "mana"), del potere divino e umano, dei comandi e dei divieti autoritari, della morte e dell'aldilà, dell'inferno, di tutto ciò che è più spaventoso della terra. Vincendo la paura, il riso ha rischiarato la coscienza dell'uomo e gli ha rivelato un mondo nuovo (sottolineatura dell'autore) (BACHTIN, 1975, p.102) 
Se consideriamo questa lettura di Bachtin sul riso medievale, possiamo capire meglio il motivo che ha spinto Boccaccio a iniziare la sua narrativa di novelle con la storia di Ser Ciappelletto che, in ultima analisi, rappresenta tutto ciò che è il rovescio della società ufficiale. Possiamo dire che questo protagonista rappresenta la rottura completa con i valori e le credenze dell'uomo medievale e, quindi, la sua storia non solo prepara il lettore all'allegria promessa dall'autore nel prologo, ma alla possibilità di rompere i ponti con tutti i valori prestabiliti e di ricominciare da capo. La prima novella del Decamenron si avvale, dunque della confessione parodica per "rischiarare la coscienza del lettore" e prepararlo al nuovo.

Due secoli dopo viene pubblicato, in Spagna, il Lazarilho de Tormes. Questo romanzo epistolare, o questa lettera confessionale, si serve della parodia come forma di denuncia sociale. È interessante osservare che, per la prima volta, l'antieroe assume la voce narrante dall'inizio alla fine del testo e lo fa da un cronotopo completamente nuovo, quello dei degradati sociali.

Il protagonista-narratore è un picaro, il corrispondente narrativo di individui reali, di emarginati sociali che abbondavano nella Spagna del Cinquecento. Il testo narra in prima persona le avventure e i mezzi poco onesti utilizzati dal picaro per sopravvivere nella società di caste che non offre possibilità di lavoro onesto. I grandi signori vivono di apparenze e non presentano soluzioni all'immobilismo sociale, anzi lo vedono come una forma conveniente di rafforzare le gerarchie e il proprio potere. Essi si appoggiano sulla politica inquisitoriale della Chiesa in Spagna, la quale difendeva fieri principi cristiani e considerava vile e basso il lavoro fatto con le mani e peccaminose le richezze da esso risultanti.

Il Lazarillo de Tormes presenta, come afferma lo studioso Mario González, una "pratica rituale sovvertita" (2005, p. 197) e fa uso della ideologia dell'Inquisizione per sconsacrarla. Lazarilho, infatti, scrive per soddisfare la richiesta di un'autorità ecclesiastica ("Vossignoria"), che vuole notizie sulla faccenda, o, a dire il vero, vuole cancellare la faccenda, per poter dichiarare che non esiste faccenda alcuna.

Il romanzo assomiglia molto alle autobiografie confessionali, la maggior parte delle quali è stata scritta da monaci e studiosi della chiesa in fin di vita, i quali, seguendo l'esempio di Sant'Agostino, lasciavano la loro testimonianza sul cammino verso l'illuminazione che credevano si potesse raggiungere solo con austerità, dedizione allo studio delle sacre scritture e grandi sacrifici. Queste autobiografie, di solito, erano rivolte ad autorità ecclesiastiche in grado di capire l'asprezza del cammino e la felicità dell'incontro mistico con il creatore.

Così, Lazarillo scrive la storia delle "asprezze della sua vita" per negare che l'arciprete, suo 
protettore, sia amante di sua moglie. Il romanzo è circolare, poiché, fin dalle prime righe, tutto converge verso le affermazioni finali, quando il picaro dimostra che è diventato un uomo per bene, perché ha un impiego statale (fa la pubblicità dei vini che si vendono in città e di altri avvenimenti d'interesse pubblico, come le condanne a morte), che, a dire il vero, è al livello più basso di tutti, ma è pur sempre un posto riconosciuto socialmente e gli garantisce una casa e, specialmente, una vita senza più fame.

Per parlare della faccenda, Lazarillo decide di cominciare la sua storia dalla nascita, raccontando la strada del proprio disonore, sempre accompagnata e determinata dall'ipocrisia delle classi dirigenti cui serve e a cui si lega per provare a superare la sua condizione di assoluta miseria. Comincia, allora, un gioco ingegnoso e parodico per provare che la verità è una questione di punto di vista.

L'ironia che fonda il testo fa capire che tutti i fatti negati dal picaro sono probabilmente reali e che, quindi, è più che ammissibile che lui abbia sposato sua moglie solo per nascondere la vera relazione di essa con l'arciprete cui serve. Parlando come uomo dabbene che è, Lazarillo garantisce a "Vossignoria" che difende il suo onore e quello di tutti i coinvolti nella faccenda, impedendo, così, che le male lingue si manifestino:

In particolare se qualcuno vuole mettermi contro mia moglie, che è la cosa al mondo che più amo, e l'amo infatti più di me stesso. Il Signore mi ha fatto non una grazia ma mille grazie a farmela incontrare, molte di più di quanto io ne meriti. E sono pronto a giurare sull'ostia consacrata che è la donna più onesta che vive entro le mura di Toledo. E se qualcuno mi viene a dire qualcosa in contrario, finisce a coltellate. In questo modo non mi dicono niente e sto in pace a casa mia. (2009, p.104)

Lazarillo garantisce che con questo discorso riesce a "tacere le male lingue" e a difendere l'onore del rappresentante della Chiesa, in modo che "Vossignoria" non si deve preoccupare della faccenda. Il racconto della propria miseria è la sua garanzia di dire la verità al destinatario della lettera, perché, dopo tanto disonore e tanti inganni che si è visto costretto a usare per sopravvivere, egli è disposto a "finire a coltellate" pur di non perdere la sua casa, il suo lavoro statale e il cibo quotidiano, una volta tanto difficile da ottenere. Il picaro è pragmatico e il suo interesse bada solo al proprio "benessere". 
Il giovane Lazarillo agli albori della sua vita era stato gettato in un mondo senza prospettive, nelle mani di un cieco mendicante e furbo, con un solo consiglio della madre "ora devi fare da solo". Così il protagonista cerca di cavarsela e di cambiare la propria situazione esistenziale a qualsiasi costo, per far parte del gruppo sociale degli uomini di rispetto, anche se nel rango più basso e raggiunto soltanto mediante la corruzione dei valori degli uomini perbene.

Vediamo che, nelle confessioni di Ser Ciappelletto e di Lazzarillo de Tormes, il racconto della propria vita non è motivato dall'intima necessità di rivelarsi e di "ri-significare" la propria esistenza, ma da una situazione momentanea, che sorge da un terzo elemento esterno che sollecita il racconto. Essi si confessano per liberare i loro pari dai problemi e, nel caso, di Lazarillo, per liberare anche se stesso.

Nella novella di Boccaccio, con la sua confessione Ser Ciappelleto salva gli amici usurai, ma garantisce anche a se stesso di morire come è vissuto, cioè, in sintonia con i "valori alla rovescia" che tanto amava e con i quali molto si dilettava. Nel Lazarillo, tutto il discorso converge nell'affermazione finale, quando egli assevera che non esiste nessuna faccenda e che avrebbe preso a coltellate chi avesse detto il contrario. Con quest'ultima affermazione il picaro cerca di garantire la sua attuale situazione, senza disturbare e senza causare problemi alla Chiesa, anche perché, garantisce Lazarillo ironicamente, sua moglie "è la donna più onesta che vive entro le mura di Toledo".

Fissati in un nuovo cronotopo, quello del "negativo", Ciappelletto e Lazarillo sono il contrappunto del mondo degli eroi e dei martiri, della dignità e delle caste, e usano i "peccati" sociali per avere "successo", perché, in una società che vive di apparenze, è necessario sembrare onesto, ma per farlo non ci vuole l'onore. Così Ciappelletto e Lazarillo sono due ribelli, ma non rivoluzionari, ad ogni modo la loro azione o parola-azione mette a nudo tutti i peccati della società in cui vivono, celebrando con il riso "la vittoria sulla paura".

Nei due testi la parodia scompone il senso del gergo confessionale e lo trasforma in arma dei protagonisti per raggiungere i loro scopi. La confessione, commenta ancora María Zambrano, “è un'azione, l'azione massima che è dato attuare con la parola" (2000, p. 45). Nel caso di Ciappelletto e Lazarillo questa parola-azione parodiata non è frutto di riflessioni interiori, ma usufrutto della parola ideologica; quella che, per Bachtin

È precisamente il prodotto della relazione reciproca tra il parlante e l'ascoltatore, tra il mittente e il destinatario. Ogni parola esprime "l'uno" in relazione all" "altro". 
Io mi do una forma verbale da un punto di vista di un altro, in definitiva dal punto di vista della comunità cui appartengo. Una parola è un ponte gettato tra me e un altro... è un territorio in comune fra il mittente e il destinatario. (apud HOLQUIST, 1999, p. 278)

La confessione parodiata fa crollare davanti agli occhi del lettore-uditore tutte le maschere sociali e i valori del mondo antico. Sorge in essa la visione di un uomo nuovo, pragmatico, che sfida tutte le convenzioni sociali e religiose e ride delle profanazioni, perché crede solo al proprio potere di trasformare le situazioni a suo favore, con la propria parola-azione.

\section{Riferimenti Bibliografici}

BACHTIN M. L'opera di Rabelais e la cultura popolare. Riso, carnevale e festa nella tradizione medioevale e rinascimentale. Torino: Einaudi, 1975.

BAKHTIN, M. Marxismo e filosofia da linguagem. São Paulo: HUCITEC, 1992.

BOCCACCIO, G. Decameron. A cura di V. Branca. Milano: Mondadori, 2001.

BOCCACCIO, G. Decamerão. Tradução Torrieri Guimarães. São Paulo: Abril, 1981.

CLARK, K., HOLQUIST, M. Michail Bachtin. Bologna: Il Mulino, 1999.

DELUMEAU, J. La paura in Occidente. Bologna: Il Mulino, 2006.

GONZÁLEZ, M. (org.) Lazarillo de Tormes (autor anônimo). Edição bilíngue. Trad. Heloísa Costa Milton e Antônio R. Esteves. Notas e estudo crítico de Mario González. São Paulo: Editora 34, 2005.

ROSSI, R. (a cura di) Lazarillo de Tormes (autor anônimo). Introduzione Manuel Vázquez Montalbán e traduzione di Rosa Rossi. Milano : Feltrinelli, 2009.

ZAMBRANO. M. La confessione come genere letterario. Torino: Pearson Paravia - Bruno Mondadori SPA, 2000. 